\title{
REAL TIME ULTRASONOGRAPHIC EVALUATION OF GYNECOLOGICAL PELVIC MASSES - A PROSPECTIVE STUDY
}

Kaushal Lovely ${ }^{1}$, Malik Rajesh².

1. RMO third year, Department of Radiodiagnosis and Imaging, Gandhi Medical College and Hamidia Hospital, Bhopal, India.

2. Associate Professor and Head, Department of Radiodiagnosis and Imaging, Gandhi Medical College and Hamidia Hospital, Bhopal, India.

\section{CORRESPONDING AUTHOR}

Dr. Lovely Kaushal,

E-2, Doctor colony,

Near hanuman mandir,

Idgah hills, Bhopal,

Madhya Pradesh- 462001.

Email-drlovelykaushal@gmail.com

\section{HOW TO CITE THIS ARTICLE:}

Kaushal Lovely, Malik Rajesh. "Real time ultrasonographic evaluation of gynecological pelvic masses - a prospective study real time ultrasonographic evaluation of gynecological pelvic masses - a prospective study". Journal of Evolution of Medical and Dental Sciences 2013; Vol2, Issue 25, June 24; Page: 4783-4791.

ABSTRACT: The space occupying lesions in female pelvis are very common over a wide age range. Many pathological conditions give rise to pelvic mass. It is difficult to arrive at an accurate diagnosis on clinical examination alone. Transabdominal and transvaginal ultrasonography are precisely helpful to determine the origin of a mass from uterus, ovaries, adnexal or extra genital structures. Serial sonographic examinations help in assessment of change in size and consistency for monitoring and for evaluation of therapeutic response. Aims: Sonographic evaluation of gynecological pelvic masses to determine the organ of origin, and correlation of sonographic diagnosis with histopathological examination. Method: A hospital based prospective study was done on 50 female patients with gynecological masses using high resolution ultrasonography and findings were correlated with histopathology examination. Result: Sonography was performed in 50 patients after a detailed clinical examination and various diagnoses were made. Patients were followed up to surgical management or serial sonographic monitoring in case of functional lesions. 52\% patients were having uterine pathologies like leiomyoma, leiomyoma with pregnancy, adenomyosis, uterine and cervical malignancies, while $46 \%$ were having adnexal pathologies like benign ovarian cyst, malignant ovarian cyst, tubo-ovarian mass, cystic teratoma etc. and rest $2 \%$ were have other pathologies like localized pus collection in the pelvic cavity etc. Accuracy to identify organ of origin was $100 \%$ in the presenting study. In the identification of the uterine pathology, $91 \%$ of fibroid diagnosed correctly by ultrasonography while uterine and cervical malignancies were diagnosed with $100 \%$ accuracy in the presenting study. In various ovarian pathologies, benign cystic ovarian lesions were detected with $100 \%$ accuracy while malignant ovarian masses and tubo-ovarian masses were diagnosed with accuracy of $80 \%$ and $85 \%$ respectively. Conclusion: Ultrasonography is highly accurate in determining the organ of origin of gynecological pelvic mass. Sonographic 


\section{ORIGINAL ARTICLE}

diagnosis of the lesion based on echogenicity, size, margins, solid or cystic or mixed nature of lesion, septation and vascularity, showing significant accuracy in correlation with histopathological diagnosis. Serial sonographic monitoring of the function lesions were helpful in the management and helps to avoid unnecessary surgical procedures.

KEY WORDS: gynecological mass, pelvis, ultrasonography.

INTRODUCTION: Ultrasonography has many advantages over the other imaging modalities like conventional X-ray, computed tomography, MRI and invasive procedures. Ultrasonography is a real time, non-invasive, safe, easy, quick tool, inexpensive, sensitive, scanning of patient involve no discomfort, results of scanning are apparent immediately on viewing screen and is a dynamic modality. Till date no hazards either immediate or late have been reported. Patients tolerate the scanning technique very well. The most important positive point of ultrasonography is that it is devoid of any radiation and this makes it the most popular imaging technique in the field of obstetrics and gynecology.

The space occupying lesions in female pelvis are very common over a wide age range. Many pathological conditions give rise to pelvic mass. It is difficult to arrive at an accurate diagnosis on clinical examination alone. Trans abdominal and transvaginal ultrasonography are precisely helpful to determine the origin of a mass from uterus, ovarian, adnexal or extra genital structures. Information about the internal anatomy \& physiology of the ovary or uterus is frequently obtained during ultrasonography that would not be evident even by direct visualization of the pelvic organs at laparoscopy or laparotomy.

Serial sonography is done to detect changes in size and appearance of a particularly monitoring of a cyst that are functional in nature, for any progressive increase in size or changes in internal components. Serial sonography is also done for assessment of change in size following therapeutic response of pelvic malignancies and ovulation timing.

In this study, we want to demonstrate the site, size and nature of pelvic masses by ultrasonography and to judge its limitations and reliability in accurately diagnosing such masses on follow up with laparotomy and histopathological examination.

MATERIALS AND METHODS: Hospital based prospective study of 50 patients was conducted from December 1984 to November 1986 on patients referred for high resolution ultrasonographic evaluation from department of Gynecology and General surgery to the Department of Radiodiagnosis and Imaging at Gandhi Medical College and Hamidia Hospital, Bhopal. The cytohistopathological diagnosis was considered as the final diagnosis. All the subjects were enrolled with detailed oral and written consents. This study was approved by ethical and scientific committee of the institute.

The patients with the masses arising namely from uterus, ovary and fallopian tubes were selected.

Ultrasonographic evaluation was performed using Network Nebula 402 ultrasound B mode gray scale sector scanner using $3.5 \mathrm{MHz}$ and $6.5 \mathrm{MHz}$ transducer. In every case, transabdominal sonography was done and in some cases finding are correlated with transvaginal sonography. 


\section{ORIGINAL ARTICLE}

In almost every case proper sonographic evaluation of uterus, endometrium, adnexa, ovaries, bladder and anterior pelvic structure, pelvic walls, cul de sac, rectum, small bowel and posterior pelvic structures was done.

Sonographic findings of each lesion were designed to assess echogenicity, shape, borders, size, composition, calcifications, septation, loculation, laterality, presence of invasion of capsule and fixation of mass. The presence or absence of ascites or other metastatic lesions were also noted in every case.

Echogenicity categories included markedly hypoechoic, isoechoic, hyperechoic and anechoic. Size was defined as the maximal dimensions of the lesion. Composition was defined as solid, cystic and mixed. Borders were defined as smooth and irregular. Calcifications were divided into those located centrally within the nodule, peripherally and none. Posterior shadowing of at least one of the suspected calcifications was required to consider the finding present. Pathological evaluation was performed on all the lesions.

RESULTS: In the present study, patients were in the range of 18 to 60 years. Majority of the patients were in the age group of 31 to 50 years with mean age of 33.8 years. Of 50 patients evaluated by ultrasonography 26 were having uterine pathologies and 23 were having ovarian pathologies. One patient was present with localized collection in to the pelvic region.

Majority of uterine lesions were fibroids (77\%) and fibroid with pregnancy (7.6\%). Adenomyosis was found in $7.6 \%$ and malignant uterine and cervical masses, each were found in $3.8 \%$ patients.

Most of ovarian lesions were benign cystic lesions (43\%) in which follicular cysts were most common (40\%), followed by luteal cyst, serous cystadenoma, mucinous cystadenoma (20\% each). Malignant ovarian masses found in $17 \%$ (4/23 of patients), of which serous cystadenocarcinoma was most common in $50 \%$ ( 2/4 of malignant ovarian masses) followed by mucinous cystadenocarcinoma and endometrial sinus tumor (25\% each). Tubo-ovarian masses were found in $26 \%(6 / 23)$ of patients with ovarian pathology. Ovarian teratoma, hydrosalpinx and ovarian torsion were found in $4.3 \%$ each.

Accuracy to identify organ of origin was $100 \%$ in the present study. In the identification of the uterine pathology, 91\% of fibroid diagnosed correctly by ultrasonography, 9\% (2/22) of fibroids were diagnosed as adenomyosis after postsurgical histopathological examination. Accuracy of ultrasonography in the diagnosis of uterine and cervical malignancies was $100 \%$ in the presenting study.

In various ovarian pathologies, benign cystic ovarian lesions were detected with $100 \%$ accuracy. Ovarian malignancies were diagnosed in 5 patients sonographically, out of which 4 diagnoses were proved correct, but 1 was corrected as ovarian torsion after postsurgical histopathological examination. Seven patients were diagnosed as tubo-ovarian mass out of which 6 were proved correctly by histopathology. One case was diagnosed as false positive and proved to be hydrosalpinx after postsurgical histopathology. One lesion of ovarian teratoma diagnosed sonographically was found to be correct by histopathology. So accuracy of diagnoses of malignant ovarian masses and tubo-ovarian masses were found $80 \%$ and $85 \%$ respectively, in present study. 
ORIGINAL ARTICLE

\begin{tabular}{|c|c|c|}
\hline TYPES OF LESION & $\begin{array}{l}\text { USG } \\
\text { DIAGNOSIS }\end{array}$ & $\begin{array}{l}\text { HISTOPATHOLOGICAL } \\
\text { DIAGNOSIS }\end{array}$ \\
\hline \multicolumn{3}{|l|}{ UTERINE } \\
\hline Fibroid & 22 & 20 \\
\hline Fibroid with pregnancy & 02 & 02 \\
\hline Adenomycosis & 00 & 02 \\
\hline Adenocarcinoma of uterus & 01 & 01 \\
\hline Carcinoma of cervix & 01 & 01 \\
\hline \multicolumn{3}{|l|}{ OVARIAN } \\
\hline Benign- follicular cyst & 04 & 04 \\
\hline Luteal cyst & 02 & 02 \\
\hline Serous cystadenoma & 02 & 02 \\
\hline Mucinous cystadenoma & 02 & 02 \\
\hline Benign cyst teratoma & 01 & 01 \\
\hline Hydrosalpinx & 00 & 01 \\
\hline Ovarian cyst torsion & 00 & 01 \\
\hline Tubo-ovarian masses & 07 & 06 \\
\hline Malignant - serous cystadenocarcinoma & 02 & 02 \\
\hline Mucinous cystadenocarcinoma & 02 & 01 \\
\hline Endometrial sinus tumor & 01 & 01 \\
\hline $\begin{array}{l}\text { Localized collection of pus in pelvic } \\
\text { region }\end{array}$ & 01 & 01 \\
\hline TOTAL & 50 & 50 \\
\hline
\end{tabular}

DISCUSSION: The present study was undertaken to evaluate the role of ultrasound in determining site, size, nature and consistency of pelvic masses and to evaluate the results of conservative management by serial sonographic examination. 50 cases were studied sonographically and histopathological confirmation of the diagnosis was obtained.

Among the uterine masses, leiomyoma was the most common tumor in the presenting study, consistent with the study of Fleischer et al ${ }^{1}$. Out of 22 cases of leiomyoma 20 cases were diagnosed correctly by sonography. Usual ultrasound findings was enlarged uterus with ill-defined areas of decreased or increased echogenicity disrupting normal fine speckled echo pattern of normal uterus. Majority of solitary leiomyomas were well defined. Degenerative changes were found in 9\% (2/22) cases.

A false diagnosis of fibroid in two cases was corrected as adenomyosis after postsurgical biopsy. Walsh et $\mathrm{al}^{6}$ described characteristics features of adenomyosis but these cases of our study only showing enlargement of uterus with normal endometrial and myometrial echotexture and without any definite mass.

According to Bezjian et al7 . Leiomyoma are one of the most common pelvic masses countered during pregnancy. We were found 2 cases of leiomyoma in the pregnant patient. These cases were showed mixed echogenic pattern. 


\section{ORIGINAL ARTICLE}

In the study of 105 patients of endometrial carcinoma, done by Requad et al ${ }^{5}$, described no ultrasound criteria were diagnostic of carcinoma, there were statistically significant difference in the uterine shape and echo pattern between stage I - II and stage III \& IV disease. 94\% of patients with stage I - II had a normal or bulbous uterus and a normal or hypoechoic parenchymal pattern with a lobular uterus and /or mixed echo pattern had stage III \& IV. Adenocarcinoma of uterus was diagnosed in only one case in our study, in which uterus was normal in size, it showed bulbar type of configuration of uterus with hypoechoic pattern and endometrial echo was prominent. Postsurgical histopathology confirmed the diagnosis as adenocarcinoma stage II.

A single case diagnosed as carcinoma cervix in which uterus was enlarged in size and cervix shows heterogeneous mass which was infiltrating in the bladder base leading to bilateral hydroureteronephrosis.

In the adnexal masses majority of ovarian lesions were benign cystic lesion (43\%) in which follicular cyst were most common (40\%), followed by luteal cyst, serous cystadenoma, mucinous cystadenoma (20\% each). All cases of follicular cysts of ovary were an incidental findings on the sonography performed for the some other reasons. The smaller cysts cannot always be differentiated from the other small fluid filled adnexal masses such as hydrosalpinx while larger cysts are similar in appearance to small serous cystadenoma and to paraovarian cysts. Serial monitoring was helpful in these cases, which shows resolution of the lesion on subsequent sonographic examination. Luteal cyst appeared as an anechoic mass with well defined walls. In our study we were found 4 follicular and 2 luteal cysts, which was consistent with the findings of Fleischer et al ${ }^{1}$.

All ovarian cystadenoma were anechoic with well defined walls. Fleischer et $\mathrm{al}^{1}$ found septation in all of their 18 cases of serous cystadenomas. We observed septation in both cases and loculations in one case. Mucinous cystadenoma may in addition contain low level echoes due to their mucin content. This finding was observed in our case. Similarly Walsh, Taylor et $\mathrm{al}^{6}$. Also found week internal echoes occasionally in cases of mucinous cystadenomas. Hence it suggests that a cystic ovarian mass with septation and internal echoes is more likely to be a mucinous cystadenoma.

A case of teratoma presented as a well defined an echoic loculated mass containing multiple dense echoes with fat fluid level was also seen in addition on turning the patient to one side. Similar findings have been described by Sandler et al. Fat fluid level considered characteristic of ovarian teratoma.

Malignant ovarian masses found in $17 \%$ (4/23 of patients), in which serous cystadenocarcinoma most common found in 50\% (2/4 of malignant ovarian masses) followed by mucinous cystadenocarcinoma and endometrial sinus tumor (25\% each). In presenting study, all malignant ovarian tumors were showing cystic mass with ill-defined walls and solid component. All cases present with ascites. R. Rosenberg et al and Morley \& Banett et al ${ }^{4}$ suggested that irregular and solid component in a cystic mass suggested gross malignant changes. None of the malignant ovarian tumor was purely cystic. In our study, 1 out of 4 malignant ovarian tumors (25\%) showed liver metastasis with ascites and peritoneal seeding. Requad et $\mathrm{al}^{5}$ demonstrate omental and peritoneal deposits in $20 \%$ of their 15 cases.

In the tubo-ovarian masses two types of patterns were seen. The first consisting of large fusiform shaped cystic masses representing fallopian tubes and second type was that of a rounded or ovoid mass with ill-defined walls. Well defined cystic tubo-ovarian masses were indistinguishable 


\section{ORIGINAL ARTICLE}

from other types of ovarian cysts, however clinical history and tenderness on physical examination helped in differential diagnosis. Ultrasound was especially helpful in cases treated conservatively since it gauged the results of treatment by serial sonographic examination.

One case of ovarian cyst, diagnosed ultrasonographically as a large anechoic cyst was found to be torsion cyst postoperatively.

Tubo-ovarian masses were found in $26 \%(6 / 23)$ of patients with ovarian pathology. Ovarian teratoma, hydrosalpinx and ovarian torsion were found in $4.3 \%$ each

Accuracy to identify organ of origin was $100 \%$ in the presenting study. Our findings were consistent with study of Fleischer et al, Lawson et $\mathrm{al}^{3}$ and Walsh et $\mathrm{al}^{6}$, reported accuracy of $91 \%, 91 \%$ and $94 \%$ respectively. In the identification of the uterine pathology, 91\% of fibroid diagnosed correctly by ultrasonography, 9\% (2/22) of fibroids were diagnosed as adenomyosis after post surgical histopathological examination. Accuracy of ultrasonography in the diagnosis of uterine and cervical malignancies was $100 \%$ in the present study.

In various ovarian pathologies, benign cystic ovarian lesions were detected with $100 \%$ accuracy while accuracy of diagnoses of malignant ovarian masses was found $80 \%$, in present study. Jain k.et $\mathrm{al}^{2}$, assessed the reliability of gray scale and color and duplex doppler ultrasound in making a specific diagnosis of malignant ovarian tumor. Results suggested that endovaginal ultrasonography enabled correct diagnosis of 38 out of 40 benign masses i.e. $95 \%$ and all malignant masses i.e.100\%. Color and duplex Doppler ultrasound enabled correct diagnosis of $82 \%$ benign masses and $78 \%$ of malignant masses.

CONCLUSION: Ultrasonography is highly accurate in determining the organ of origin of gynecological pelvic mass. Sonographic diagnosis of the lesion based on echogenicity, size, margins, solid or cystic or mixed nature of lesion, septation and vascularity, showing significant accuracy in correlation with histopathological diagnosis. Serial sonographic monitoring of the function lesions were helpful in the management and helps to avoid unnecessary surgical procedures. Hence sonography is real time, non-invasive, safe, easy, quick, devoid of any radiation hazard and high accuracy; it must be use first line modality for the evaluation of gynecological pathologies.

\section{REFERENCES:}

1. Fleischer A.C: differential diagnosis of pelvic masses by gray scale sonography. Am. Jr. of Roentology.1978; 131:469-76.

2. Jain K.A: Prospective evaluation of adnexal masses with endovaginal gray scale and duplex and color Doppler ultrasound; correlation with pathologic findings. Radio.1994; 191:63-67.

3. Lawson T.L. et al: Ectopic pregnancy criteria and accuracy of ultrasound diagnosis. Am. J. roentgenol.1978; 131:153-56.

4. Morley E. et al: The use of ultrasound in the diagnosis of pelvic masses. Br. J. Radiol.1986; $43 ; 602-616$.

5. Requad C.K., Wicks J. D: ultrasonographic in the staging of endometrial adenocarcinoma. Jr. of Radiology. 1981; 140; 781-785.

6. Walsh J.W., Taylor K.J.W: Gray scale ultrasound in 204 proved gynecologic masses; accuracy and specific diagnostic criteria. Jr. of Radiology. 1979; 130; 391-397\&132; 87-90. 


\section{ORIGINAL ARTICLE}

7. Bezian A, Carretero M: ultrasonic evaluation pelvic masses in pregnancy. Clin. Obstet. Gynae. 1977; 20; 325-38.

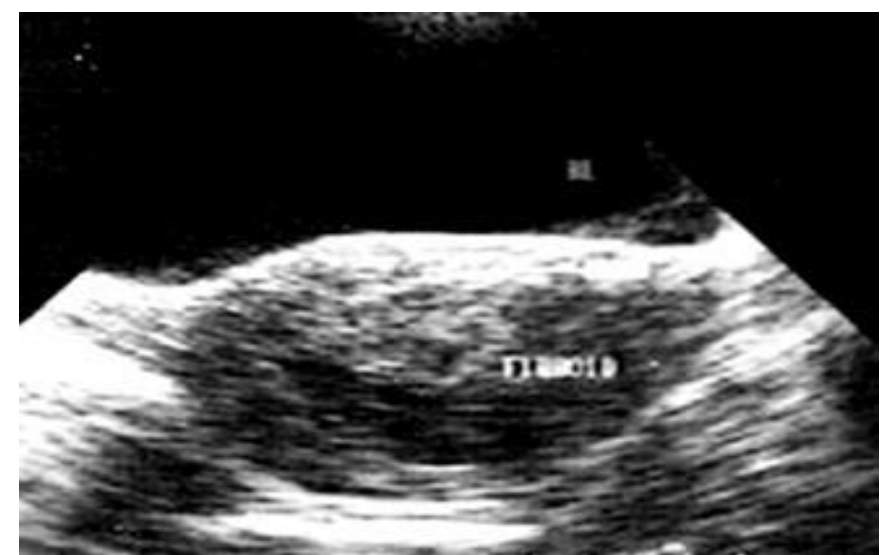

FIGURE 1: Hypoechoic fibroid in posterior wall of uterus displacing the endometrial echo complex

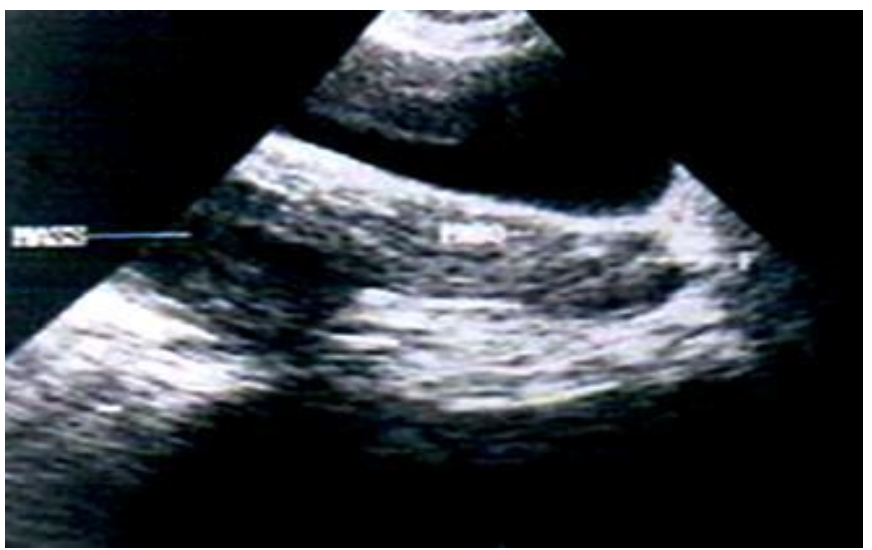

FIGURE 2: Multiple hyper and hypoechoic area in posterior wall of uterus - multiple fibroids

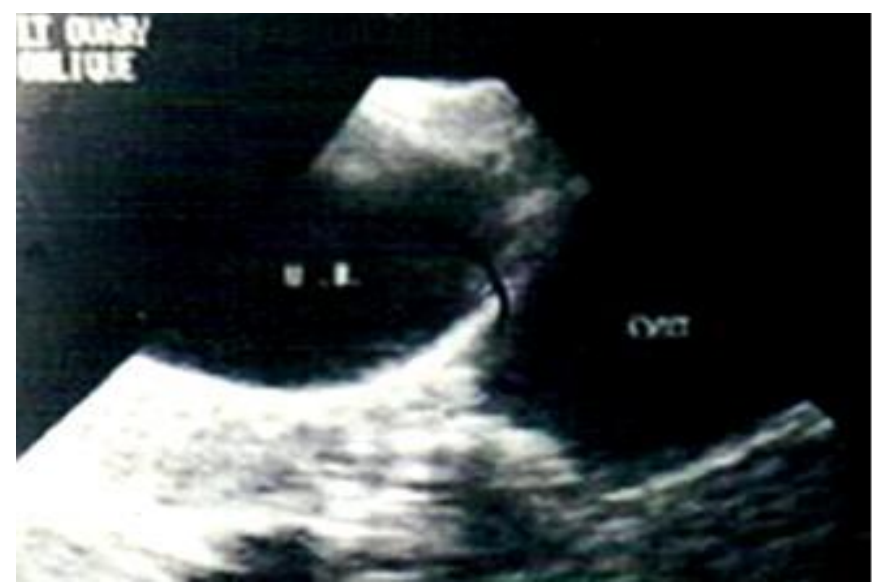

FIGURE 3: A well defined smooth walled ovarian cyst left side- serous cystadenoma. 


\section{ORIGINAL ARTICLE}

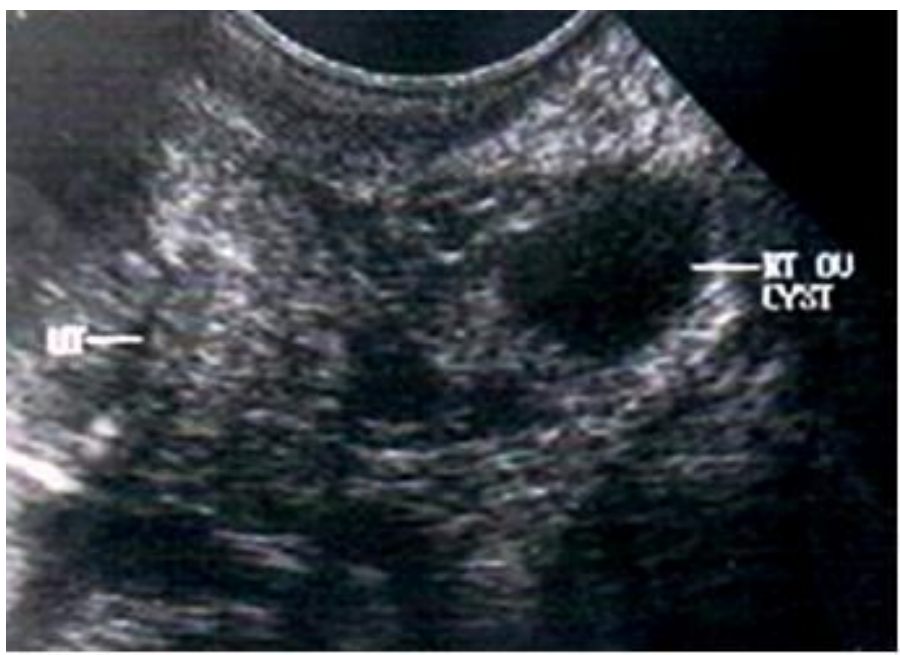

FIGURE 4: Adnexa shows area with septations and echogenic contents (hemorrhage) - corpus luteal cyst

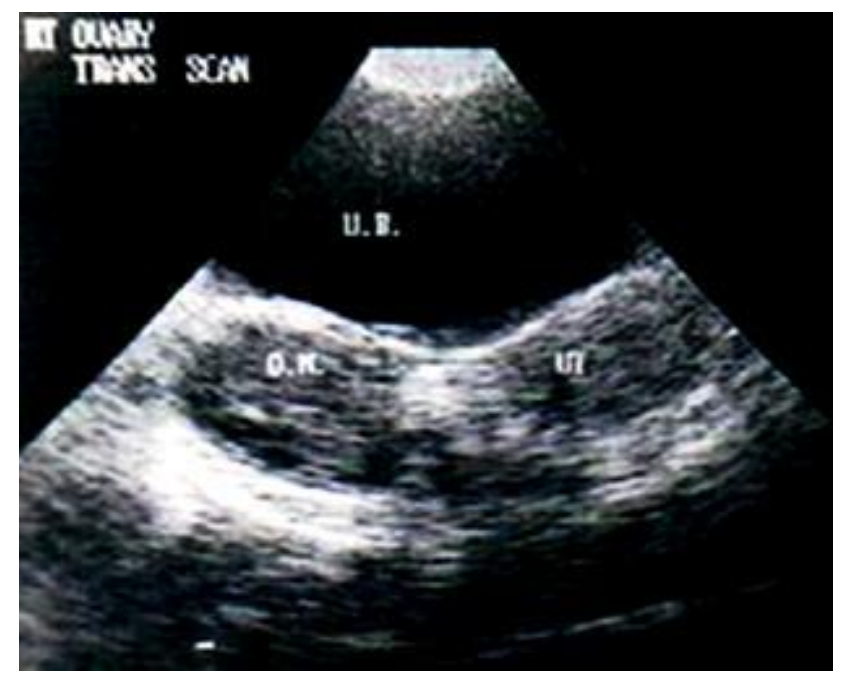

FIGURE 5: Right adnexal showing a heterogeneous mass - Tubo-ovarian mass

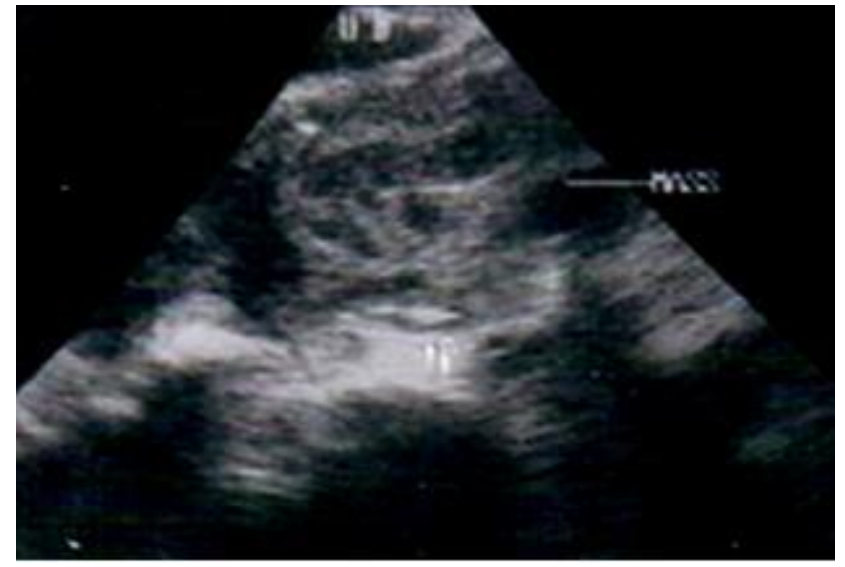

FIGURE 6: Left ovary shows large heterogeneous mass with multiple septation and solid components - cystadenocarcinoma 


\section{ORIGINAL ARTICLE}

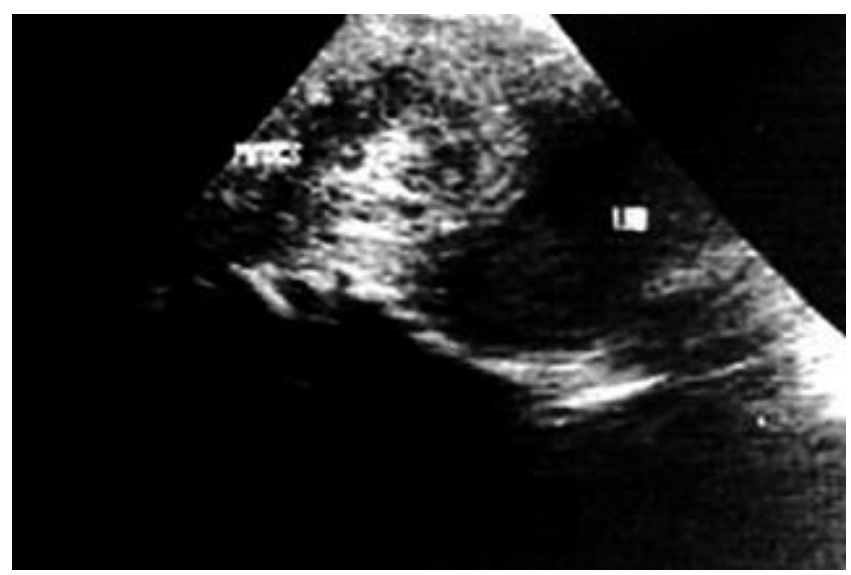

FIGURE 7: Mass of heterogeneous echotexture in fundus of uterus - large degenerating fibroid

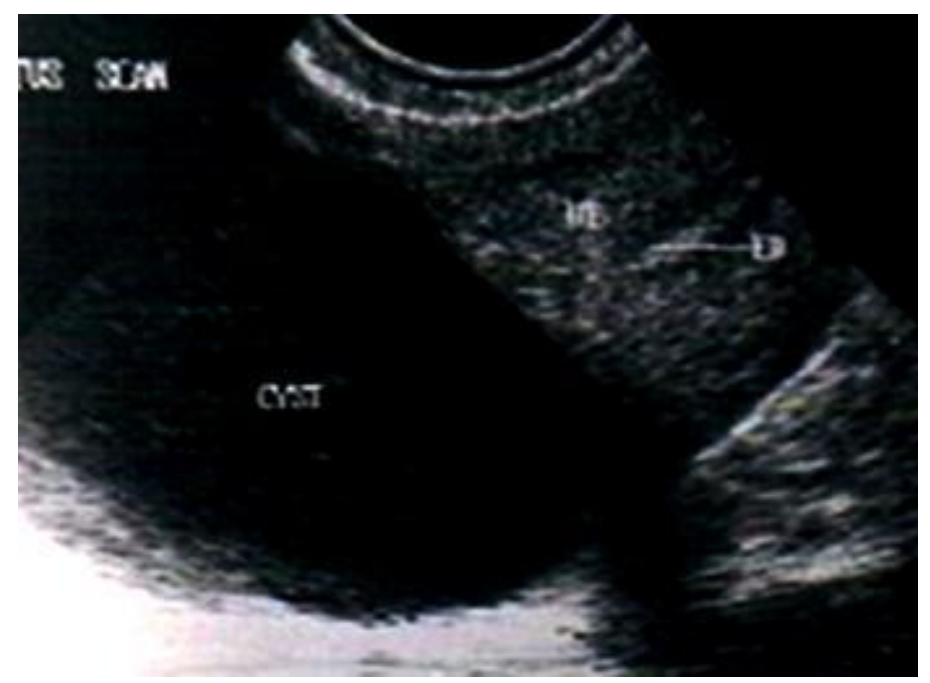

FIGURE 8: Left ovary shows anechoic cyst with no septation or any internal echoes - serous cystadenoma 